\title{
A Study of The Morpheme Meaning Variation of Word-formation Based on the New Abbreviations in Contemporary Chinese
}

\author{
Qi Cao1, a*and Yuanyuan Ma², b \\ 1, 2International Exchange College, Bohai University, Jinzhou, Liaoning province of China \\ aqicao2008@126.com, b2723044706@qq.com
}

Keywords: Abbreviations; Word morphemes; Variation

\begin{abstract}
Driven by the economy principle of language, a large number of new abbreviated words have emerged in Contemporary Chinese. With the high frequency of new abbreviations, morpheme meaning of abbreviations produce various variation phenomenon. The morpheme variations mainly increase some new morphemes and the new meaning of morpheme for the original morphemes in Chinese system. The morphemes of abbreviations have strong dependence on the corresponding original phrases, and some morpheme meanings of abbreviated words are multidimensional and temporary. This paper clarifies the morphemes meaning relations of shortened words. It has important reference value for Chinese language teaching and compilation of Chinese dictionaries.
\end{abstract}

\section{Introduction}

With the continous deveiopment of society, new things and new ideas emerge in endlessly, so the language as a communication tool generates a large number of new words and phrases. Among them , some new words have long syllable structure, stable structure and high frequency of use , such as the word "aolinpikeyundonghui". In language application, phrases with long syllable structure and high frequency of use are obviously not conducive to the convenience and efficienty of expression. Therefore, driven by the economic principle of saving time and effort, a large number of abbreviation new words are produced. The emergence of a large number of abbreviations is the result of the change of coding mechanism under the promotion of language economic principle. With the further lexicalization of acronyms, the corresponding relationship between acronyms and original phrases is gradually blurred. So it is more necessary to explore the influence of acronyms on morpheme meaning variation. The researh in this aspect will also be conducive to the further development of Chinese vocabulary teaching and research.

\section{The Relationship between Chinese Abbreviation form and Morpheme Meaning}

Abbreviations in Chinese is a special lexical phenomenon, which is not formed by morphemes directly, but by abbreviation of long phrase. Although words with longer syllable tend to have more complex and precise meanings, they are not conducive to the convenience of communication. Therefore, the more frequently words are used, the eariser it is to produce abbreviations. Such as "aolinpikeyundonghui" is abbreviated as "aoyun", "boshishengdaoshi" is abbreviated as "bodao", "shifandaxue" is abbreviated as "shida" and ect. The frequency use of these words within a certain range are very high, and the abbreviations have few syllables, but it is equivalent to the meaning of the original phrase. It is obviously more economical and convenient.

Chinese abbreviations are mainly produced by truncation, digital generation and extraction. [1] The variation of morpheme meaning of abbreviations from different sources is different. The meaning of word morphemes in abbrevitaions comes from the meaning of corresponding words in the original phrase. Some word morphemes are consistent with the original morphemes of homophones in Chinese, and some of them are different or even completely different.

Truncation truncates a part of the original phrase, for example, "FuDan university"is abbreviated as "FuDan". The structure of the original phrase is "word 1plus word2", and the form of abbreviation is "word1", but the "word1" after abbreviation represents the meaning of the whole phrase, which is different from the "word1" in the original phrase. However, the morphemes of the 
two do not change.

Digital abbreviation is a combination of numbers and the characteristic morphemes associated with the original phrase. For example , "tonghang" , "tongyou" and "tongshang" are abbreviated as "santong". It is composed of numbers and common morphemes. The word "tong" in this abbreviation can refer to any word in the original phrase, so it is hard to solidify the meaning of the word "tong". There is also another numerical abbreviation, for example, "sour" , "sweet" ,"bitter" and "salty" are summarized as "five flavors". As we can see, there is no common morpheme in the original phrase, the abbreviation is made up of numbers and morphemes that represent common semantic featrues. The word "flavor" in abbreviation is consistent with the basic meaning of the original morpheme, and there is no variation.

The above two forms of abbreviations have not changed the base morpheme meaning. The most worth studying abbrevitaion is the extraction abbreviation. The extraction abbreviation is formed from the recombination of some morphemes extracted from the original phrase, such as the word "foreign capital company" is abbreviated as "foreign company". Abberaviations used at high frequencies are gradually lexicalized, with clear meaning and fixed form. People no longer use the original phrase form of abbreviations to understand their meaning. For instance, abbreviations like "chaoshi", "zhishang", "yingxing" are actually the lexicalizations of the corresponding phrase "chaojishichang", "zhilishangshu" and "dianyingmmingxing" after abbreviation. These abbreviations have become close -spaced two-syllable words. Such extracted abbreviations may cause some morpheme variations due to the recombination of their constituent components, for example, in the seventh edition Modern Chinese Dictionary, only list one definition of "qin":stand with your heels up, now used as a sign of hope. But in abbreviation word "waiqi", it can only be understand as "company". As the morphem "qi" is used as "company" frequently, The morpheme"qin"adds a new meaning. Therefore, the discussion about the morpheme variation in Chinese produced by abbreviation new words is very important. It can provide useful reference for compiling Chinese dictionary and teaching Chinese language.

\section{The Morpheme Variation Types of New Abbreviations}

The production and high frequency use of abbreviated new words change the morpheme of contemporary Chinese, that is, produce morpheme variation. The morpheme variation is manifested in two cases, namely, the addition of new morpheme to the Chinese system and the addtion of new menaings to the original morpheme.

Add New Morphemes to the Chinese System. After re-analysis of the abbreviated phrase, the morpheme meaning of the phrase has some change, and some morphemes have no relation with the oringnal morpheme meaning. For example, in the abbreviations "guoqi", "siqi", "waiqi", etc, the word "qi" has a new meaning --company, which is not related to the oringinal meaning. the relation between the two meaning is old and new homophones. Such morpheme variation adds new morpheme to contemporary Chinese. In contemporary Chinese, there are fewer new morphemes produced by abbreviation, but most of them add new morpheme meaning to the original morpheme of Chinese.

Add New Morphemes to the Original Morphemes. The varition of the morpheme meaning of some abbreviated new words in contemporary Chinese is an extension on the basis of the oringinalmorpheme meaning, which only adds a morpheme meaning to the original morpheme. For example, with the development of society, the word "international internet" come into being, for the sake of convenience, the term "wangluo" is often used to refer specially to the word "international internet". In daliy work and life, a large number of new words are formed by the word "wangluo", such as "wangluogouwu", "wangluoyouxi", "wangluodianhua", "wangluowenxue", "wangluoyingxiao", "wangluoaiqing", "wangluoanquan", "wangluodaxue", "wangluoguanggao", "wangluojingji", "wangluoqinquan", "wangluoxinwen" and ect. Frequently used phrases produce acronyms, such as "wanggou", "wangyou", "wanghua" and so on. the word "wang" in abbrevaitions directly carries the meaning "internet". Clearly, the new morpheme meaning of "wang" has an extend relationship with its original meaning "organizations or systems that crisscross like a web". 
But the new morpheme meaning is more accurate and specific.

For another example, the common meaning of "ban"in Chinese is "to deal with , to handle", "ban" is used as a verb. The word"bangongshi" represents the place where business is handled. Such as proper nouns "jihuashengyu bangongshi", "zhaosheng bangongshi”, "qiaowu bangongshi”" and ect. So the word "bangongshi" is further extended to meaning "a department dealing with or handling certain matters". With the high frequency of the use of these departments in the society, "jishengban", "zhaoban", "qiaoban" and other relevant abbreviations have emerged. Apparently, "ban" in the abbreviations carries the meaning of "bangongshi". In the fifth edition of the Modern Chinese Dictionary published by the Commercial Press in 2005, only four meanings of "ban" used as verbs were listed:handle;create and oprate;purchase and arrange;punish. BY2014, the sixth edition of the Mordern Chinese Dictionary had added a fifth meaning on the basis of four original meaning---office, party office or college office. There are some new morpheme meanings similar to "ban" in contemporary Chinese, which may not be included in the normative dictionary due to its temporary and unstable nature.

\section{The Multidimensional and Temporary Variation of the Morpheme Meaning of Abbreviated Words}

There are many abbreviations with the same morpheme in Chinese abbreviations, but the meanings of the same morpheme in different abbreviations are not always the same. For example, "shuodao" and "biandao", the word "dao" in "shuodao" means "tutor", the word "dao" in "biandao" means "director". It should be noted that in the Modern Chinese Dictionary, there are five meanings about "dao":guide; conduction; enlighten; director; surname. The meaning "tutor" does not show up directly, and the fourth meaning of "dao" shows the meaning "director", however, from the examples given, it only reflects the use of "dao" as a verb. Actually, the word "dao" is used more frequently in nominal and often combined with the surname in the form of " $x$ dao", such as "fengdao", "zhangdao", "wudao" and so on. One of the direct cause of the polysemy of the same morpheme is that abbreviations come from different sources and carry different meaningsin different abbreviations. They bring new morpheme meaning to the original morpheme of Chinese

The morphemes of abbreviations are strongly dependent on the corresponding original phrase. Due to the different components of the corresponding original phrase, the morphemes of homophones morphemes in different abbreviations may carry different morpheme meanings, so the morphemes of some abbreviations often have various crossover states. For example, "caisedianshiji" abbreviated as "caidian", and the word "dian" means "television"; while "jiayongdianqi" abbreviated as "jiadian", the word "dian" means "electrical appliances"; when an aspiring actor who come into contact with movies, tv series for the first time, it produced the new word "chudian". The word "dian" in "chudian" means "movies or tv series", which is far away from the original meaning "electricity". therefore, the understanding of acronyms and their morpheme meaning has a strong dependence on the original phrase, and the corresponding to the abbreviations has become the premise of using and understanding abbreviations. [2]

Some of the new morpheme meanings in Chinese abbreviations have temporary characteristics. For example, the Chinese word " $x$ zhang" for official position comes after a person's last name, people always skip the word "zhang" and call it " $\mathrm{X}$ " for short. For instance, "zhangsanjuzhang" is generally called as "zhangjuzhang", collegues at lower or lower levels within the system and other familiar people can further abbreviated it as "zhangju". Similar official postions, such as "buzhang", "tingzhang", "xiaozhang", “yuanzhang", "xianzhang", "chuzhang", "kezhang", "duizhang", "banzhang"can also be abbreviated as the form "surname plus X". Of course, the positions like "zongjingli", "zonggongchengshi" because of the long syllables, are more easily abbreviated as the form "surname plus zong". However, due to their narrow scope of use, they are generally used in informal occasions. They are only temporarily combined with a surname to form an appellation word, which is temporary and can not become a new morpheme meaning. [3] The morphemes in abbreviations have a new morpheme meaning temporarily. While the morphemes formed in abbreviations have new morpheme meaning and become economical and convenient, it also makes 
it difficult for people to understand the meaning of abbreviations accurately. Especially during the international Chinese teaching, the multidimensional nature of the morpheme meaning in abbreviations will undoubtedly confuse the learners who learn Chinese as a second language. Therefore, it is of great value for Chinese language teaching and compilation of Chinese directionaries to study the chararctristics of abbreviated words in contemporary Chinese and to clarify the meaning relationship between abbreviated words abd morphemes.

\section{Acknowledgment}

The research is supported by the Liaoning Provincial Social Science Fund Project " A study of Chinese constructional teaching based on interlanguage corpus for two language acquisition "(L18AYY001)

\section{References}

[1] Z. P. Yin: Methods and Principles For Constructing Abbreviations[J]. Language Teaching and Reserch, 1999. (2), p75. (In Chinese)

[2] G. Q. Xu: The Semantic Relation Between Abbreviations and Original Words[J]. Chinese Constrction, 1998, (12), p28. (In Chinese)

[3] Q. Cao: Investigation and Ananlysis of Chinese New Morpheme in the New Peried [J]. Language Application, 2012, (4), p15. (In Chinese) 